\title{
MEDICIÓN DE LA INJUSTICIA AMBIENTAL SOBRE POBLACIONES VULNERABLES Y CARENCIADAS POR ANEGAMIENTOS PLUVIALES: UN ANÁLISIS EN SANTA FE DE LA VERA CRUZ (ARGENTINA) BASADO EN SIG
}

\author{
Andrea Bosisio \\ Facultad de Ingeniería y Ciencias Hídricas, Universidad Nacional del Litoral, Argentina \\ acbosisio@yahoo.com.ar \\ ORCID iD: https://orcid.org/0000-0002-3962-8618 \\ Antonio Moreno Jiménez \\ Facultad de Filosofía y Letras, Universidad Autónoma de Madrid, España \\ antonio.moreno@uam.es \\ ORCID iD: https://orcid.org/0000-0001-6687-1448
}

Recibido: 06/03/2019; Aceptado: 01/07/2019.

Cómo citar este artículo/Citation: Bosisio, A. y Moreno Jiménez, A. (2019). Medición de la injusticia ambiental sobre poblaciones vulnerables y carenciadas por anegamientos pluviales: Un análisis en Santa Fe de la Vera Cruz (Argentina) basado en SIG. Estudios Geográficos, 80 (287), e020. https://doi.org/10.3989/estgeogr.201937.017

RESUMEN: Los anegamientos producidos por lluvias intensas, recurrentes en muchas ciudades emplazadas dentro de planicies aluviales y con deficiente escorrentía, suelen ocasionar dispares grados de afección en la población, susceptibles de valorar desde la emergente perspectiva de la justicia ambiental. En esta aportación se pretende evaluar la exposición diferencial a dos eventos de anegamiento hídrico-pluvial por parte de varios grupos sociales vulnerables y con privaciones en la ciudad de Santa Fe de la Vera Cruz (Argentina) para desvelar posibles injusticias. Metodológicamente se delimitó el área urbana poblada (AUP), como ámbito relevante, y se seleccionó un conjunto de indicadores sociodemográficos expresando vulnerabilidad y privaciones humanas. El análisis recurrió intensamente a la tecnología SIG y a la técnica estadística de la $\chi^{2}$, para estimar, como resultados relevantes, qué grupos sociodemográficos y qué partes de la ciudad han sufrido una sobre-afección calificable de injusta. La confrontación con los hallazgos de otros autores permitió reivindicar una mejor exactitud de nuestros resultados para el caso estudiado y postular la idoneidad y utilidad de la metodología adoptada en la planificación y gestión urbana, al considerar unas injusticias ambientales largamente soslayadas.

PALABRAS CLAVE: Sistemas de Información Geográfica (SIG); poblaciones vulnerables; anegamientos; justicia ambiental; Santa Fe.

\section{MEASURING ENVIRONMENTAL INJUSTICE ON VULNERABLE AND DEPRIVED POPULATIONS CAUSED BY PLUVIAL WATERLOGGING: A GIS-BASED ANALYSIS IN SANTA FE DE LA VERA CRUZ (ARGENTINA)}

ABSTRACT: Waterlogging caused by heavy rains, a recurring problem in cities located within floodplains having poor runoff, often results in disparate degrees of the population' affection, which can be assessed from the emerging perspective of environmental justice. This contribution addresses to assess the unequal exposure of various vulnerable and deprived social groups to two events of water-pluvial flooding in the city of Santa Fe de la Vera Cruz (Argentina), to uncover possible injustices. Methodologically, a specific "urban populated area" is adopted as relevant area, and a set of sociodemographic indicators, denoting human vulnerability and deprivation, is selected. The analysis has intensely relied on GIS and the $\chi^{2}$ statistical technique to estimate which sociodemographic groups and what parts of the city have suffered from unjust over-exposure. The comparison to other authors' findings has enabled to assert the better accuracy of our results in the case studied, and to claim for the adequacy and usefulness of the method for urban management and planning, taking into account some largely disregarded environmental inequities.

KEY WORDS: Geographic Information Systems (GIS); vulnerable people; waterlogging; environmental justice; Santa Fe. 


\section{INTRODUCCIÓN}

Desde fines del siglo XX, el concepto de justicia ambiental (JA) está aportando un marco propicio para la evaluación y cuantificación de desigualdades sociales, diagnosticándolas desde el punto de vista geoambiental. Tales injusticias se entienden, en términos distributivos, como situaciones en las que ciertos grupos de población soportan una "carga" (peligro, afección) ambiental desproporcionada $y$, por tanto, injusta (Towers, 2000; Walker, 2012; Chakraborty, 2017). A menudo el foco se ha puesto en categorías demográficas desfavorecidas, tales como las minorías raciales o étnicas, ancianos, personas vulnerables (Schneiderbauer et al., 2017), pobres, carenciadas, etc.

Los estudios al respecto han afrontado el importante reto de clarificar conceptos básicos - e intentar medirlos - tales como los de vulnerabilidad (e.g. Cutter et al. , 2000 y 2003; Birkmann, 2006; Egea Jiménez et al., 2012; Palacios García et al. 2018), riesgos (e.g. Blaikie et al., 1994), privaciones (Townsend, 1987), etc. Así mismo, otro punto delicado al respecto ha sido dilucidar una metodología efectiva para clarificar cuánta desigualdad hay, esto es, qué grupo sociodemográfico soporta más afección y cuánta más (o menos). Los trabajos de Moreno-Jiménez (2010) y Maguire y Sheriff (2011) han propuesto soluciones técnicas en ese sentido.

En los últimos años también, el análisis de las diferencias socioeconómicas entre la población expuesta a potenciales inundaciones ha desarrollado variadas metodologías de cuantificación, considerando no solo la vulnerabilidad, sino también la resiliencia poblacional (Walker y Burningham, 2011; Rufat et al., 2015; Sarma et al., 2016; Swim y Bloodhart, 2018; Atuesta Montes et al., 2018). Utilizando tanto los SIG, como técnicas estadísticas, se obtienen y brindan mapas de riesgo que evidencian patrones de disparidad socioeconómica en las zonas afectadas, de gran utilidad en el momento de realizar la gestión y ordenación de estas áreas (Török, 2018; Qiang, 2019). Así mismo, se ha abordado la caracterización de las circunstancias y el entorno de ciertos grupos vulnerables ante inundaciones, como han hecho Sánchez González y Chávez Alvarado (2016) para los ancianos.

Los anegamientos en localidades provocados por Iluvias intensas revisten gran importancia y las vulnerabilidades asociadas están siendo estudiadas en diversas zonas del mundo (e.g. Pandey et al., 2010; Sultana, 2010; Hsu et al., 2011); en América Latina, han sido abordados analíticamente por varios auto- res (e.g. Tucci, 2007; Aragón-Durand, 2014; Paoli et al., 2015). Sus causas principales responden, junto a unas lluvias torrenciales, a la reducción de la capacidad de infiltración y retención de los excesos hídricos, provocadas por obras que refieren a una urbanización creciente y que modifican el uso de la tierra (Aragón-Durand, 2014, p. 31). Esto es observable en diversas ciudades latinoamericanas, en las cuales estos procesos se asocian a un aumento considerable de población en la periferia y a una urbanización espontánea no regulada que se genera principalmente en áreas marginales con cotas bajas y susceptibles de inundaciones, como sucede en las planicies aluviales (Tucci, 2007, p. 42).

La preocupación por este problema, a la vez social y ambiental, ha motivado la presente investigación en la que se avista evaluar, de manera sistemática y estandarizada, el grado de inequidad ambiental entre distintos grupos sociodemográficos y zonas en la ciudad de Santa Fe, derivado de los anegamientos por lluvias acaecidos en años recientes. A tal fin se ha concebido un novedoso análisis cuantitativo para estimar la afección diferencial ocurrida. Específicamente se pretende dar respuesta a los siguientes cuestionamientos:

- ¿En el conjunto de la ciudad, los grupos carenciados y vulnerables han estado más o menos expuestos a los anegamientos acontecidos que la población total? ¿En qué cuantía? ¿Cabe sostener la existencia de injusticia ambiental en Santa Fe por ese motivo?

- ¿Qué variaciones intraurbanas existen en las cifras de población afectada por esos anegamientos? ¿Afloran diferencias importantes calificables de no equitativas? En tal caso ¿dónde y en qué grado?

- ¿Qué diferencias temporales emergen al respecto entre los anegamientos de 2010 y 2015 ? ¿En qué lugares se han mitigado los impactos?

Por tanto, se avista como objetivo general determinar, mediante la implementación de diversos procedimientos de análisis espacial soportados con sistemas de información geográfica, la desigual exposición de diferentes grupos vulnerables o con privaciones, no a las amenazas, sino a los serios eventos hídrico-pluviales acaecidos en la ciudad de Santa Fe durante los meses de marzo de 2010 y marzo de 2015. Con ello se trata de ilustrar un modo de diagnosticar cuantitativamente injusticias ambientales de forma asequible y replicable. Los hallazgos se espera que resulten, metodológica y 
empíricamente, de utilidad tanto para desvelar algunas elusivas, pero flagrantes injusticias urbanas, como para propiciar políticas paliativas de las mismas.

\section{FUENTES Y METODOLOGÍA}

La ciudad de Santa Fe de la Vera Cruz se encuentra emplazada en la llanura de inundación de dos grandes ríos latinoamericanos, el Paraná en el este y el Salado al oeste, lo cual resulta en niveles topográficos muy bajos y terrenos anegadizos. Dada la estructura de la ciudad, y con el fin de centrarnos en el área más significativa, se delimitó como ámbito estricto de estudio el "área urbana poblada (AUP)", la cual excluye los espacios no residenciales y periféricos no urbanizados. Con el software ArcGIS 10.3 se procedió a realizar la delimitación vectorial de dicha área basándose en la interpretación de imágenes aéreas y de la cartografía de usos del suelo, incluyéndose zonas de uso residencial, comercial y ocio, donde la presencia diaria de población es importante (Figura 1).

FIGURA 1

LÍMITE MUNICIPAL DE LA CIUDAD DE SANTA FE DE LA VERA CRUZ Y DEMARCACIÓN DEL ÁREA URBANA POBLADA, AUP

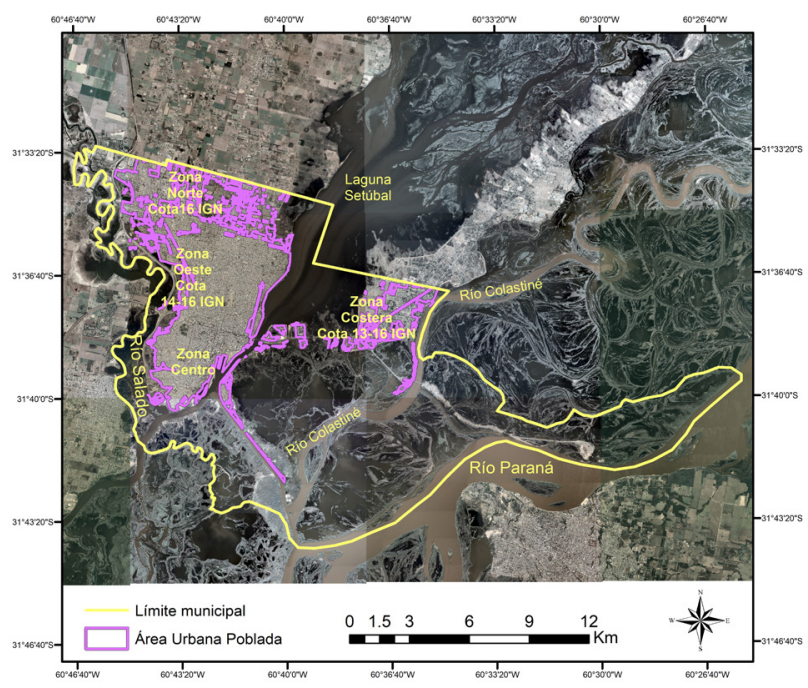

Fuente: elaboración propia.

Con el fin de obtener resultados adecuados para la valoración pública y la eventual intervención ulterior, se han adoptado dos niveles de análisis, considerando en primer término el conjunto de la ciudad (AUP), y luego la afección en los ocho distritos administrativos (Figura 2). Teniendo en cuenta las posibles utilidades cognitivas o aplicadas de los resultados, los análisis realizados para esos niveles de partición espacial no han sido idénticos en el método, ni tampoco en cuan- to a desagregación de la población vulnerable y con privaciones. Oportunamente se irán concretando tales extremos en aras de la claridad y comprensión de las decisiones adoptadas.

Se decidió adoptar una metodología que evaluase sistemáticamente las posibles injusticias ambientales para un selecto conjunto de grupos sociodemográficos en la ciudad. A tal fin se ha recurrido a un enfoque estadístico inferencial mediante el que se trata de confrontar la exposición a los anegamientos de ciertos grupos desfavorecidos, bien por su condición de vulnerables, bien por sufrir privaciones o carencias, con la exposición observada en una población o universo de referencia (e.g. algún total, como el de la población) que se propone como válido o razonable (científica o socialmente). Tal cotejo lógico habilita para una valoración de los resultados mejor fundada, transparente y contrastable. Las variables o indicadores seleccionados, tanto para la dimensión de vulnerabilidad como para la de privación (Tabla 1), fueron considerados en función de su disponibilidad y adecuada cuantificación en el último censo poblacional llevado a cabo en Argentina en el año 2010 (INDEC, 2013), ya que aporta la base informativa más amplia, consistente y estandarizada. Así mismo cabe advertir que otras variables significativas, como por ejemplo discapacidad, ingresos, etc., usualmente consideradas en este tipo de análisis, no pudieron ser incluidas en el presente trabajo debido a la carencia de tales datos estadísticos.

La lógica de nuestro análisis se podría resumir así: si se asume que la distribución espacial de un grupo sociodemográfico dado es similar a la de la población total, su grado de afección por anegamiento sería también análogo al de esa población de referencia; pero si aquélla fuese distinta, entonces podría estar en una situación / exposición más perjudicada o más favorecida, comparativamente hablando. Bajo tales premisas las hipótesis planteadas aquí, adoptando los niveles de confianza utilizados habitualmente (e.g. $\alpha=0,01$ o $\alpha=0,05$ ), serían:

$H_{0}=$ no hay diferencia significativa entre la proporción de exposición a anegamiento de ese grupo sociodemográfico y la del total de la población en la ciudad.

$H_{1}=$ hay desigualdad estadísticamente significativa en la afección observada en la población y en la muestra (cada uno de los grupos analizados), lo que se derivaría de que ambas exhiben una distribución espacial tan distinta que no puede imputarse al azar, sino a procesos urbanos definidos, que podrían acarrear injusticias ambientales. 


\section{Datos}

La base de geodatos digital utilizada para la obtención de los indicadores mediante geoprocesamiento y técnicas estadísticas se construyó en el SIG a partir de información procedente de diversas fuentes:

- Datos estadísticos poblacionales registrados para 399 radios censales urbanos de la ciudad de Santa Fe, del último Censo Nacional de Población y Vivienda 2010 (INDEC, 2013). De ellos se escogieron 10 indicadores sociodemográficos de vulnerabilidad y privaciones sociales (Tabla 1). Procede añadir que los radios censales (RC) urbanos son las unidades estadísticoespaciales mínimas del censo y que contienen habitualmente unas 300 viviendas.

- Una ortofotografía aérea de la ciudad de Santa Fe de septiembre de 2013, cedida por el Instituto Geográfico Nacional de la República Argentina (IGN), con resolución de 1,20 metros.

- Capas vectoriales con límites de distritos administrativos y áreas urbanas anegadas por lluvias intensas suministradas por la Municipalidad de la ciudad de Santa Fe (MCSF), con datos relevados en marzo de 2010 y 2015 (Figuras 3 y 4).

FIGURA 2

DELIMITACIÓN DE LOS DISTRITOS ADMINISTRATIVOS SUBDIVIDIDOS EN RADIOS CENSALES EN LA CIUDAD DE SANTA FE DENTRO DEL AUP

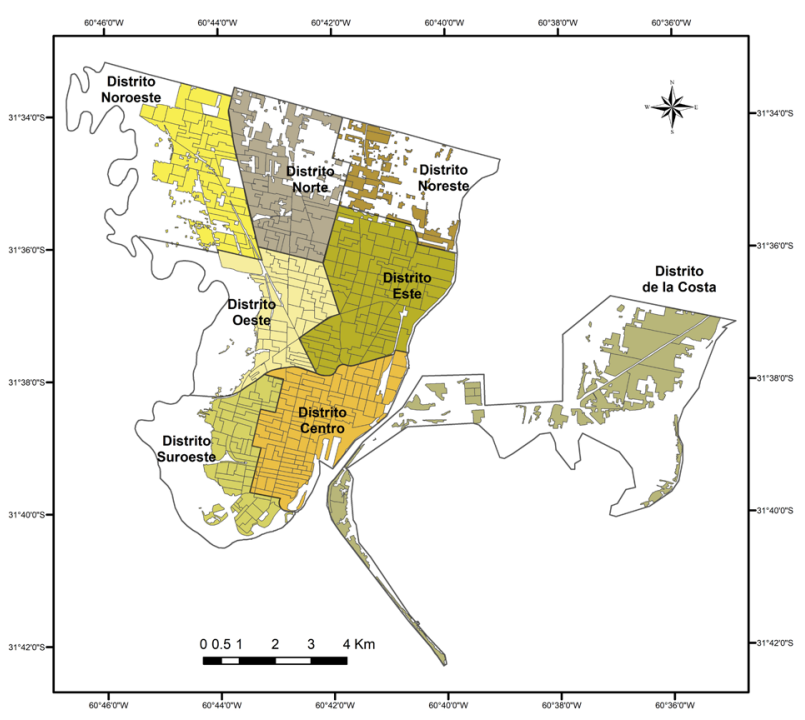

Fuente: elaboración propia.

\section{Métodos}

Como en otros estudios similares basados en SIG, el método adoptado para determinar injusticias ambientales implicó realizar estimaciones de resultados intermedios, partiendo de capas vectoriales que contenían la información sociodemográfica y de anegamientos. A tal fin se ejecutaron varios geoprocesos (unión, intersección y corte de las capas vectoriales), mediante el software ArcGIS 10.3. Como primer paso se realizó una unión espacial entre las dos capas que contenían la información estadística demográfica de los grupos de interés y del total poblacional por radio censal y las zonas anegadas, durante ambos eventos hídrico-pluviales, tanto para el año 2010, como para el 2015 separadamente.

En la capa resultante de esta unión fue posible calcular, con técnicas de geoprocesamiento estadístico, la superficie y población afectada total y para cada grupo sociodemográfico seleccionado, en diferentes campos creados para tal fin, y usando la proporción de la superficie anegada de cada radio censal como estimador de la población expuesta/ afectada.

Con el objeto de diagnosticar las desigualdades intraurbanas debidas a la afección de anegamientos (ocasionados por lluvias intensas) la hipótesis de inequidad fue testeada mediante dos técnicas complementarias: el test de bondad de ajuste mediante la prueba $\chi^{2}$ (Siegel, 1976, p. 64-69; Mendenhall et al., 2010, p. 595-597), y la balanza de la justicia ambiental descriptas en Moreno Jiménez $(2010,2013)$ y Moreno Jiménez et al. (2014a; 2014b; 2016, p.121; 2017).

La primera de estas técnicas es un test de independencia ampliamente conocido, que tiene por finalidad confirmar la hipótesis nula $\left(\mathrm{H}_{0}\right)$, la cual plantea que la probabilidad de exposición de un determinado grupo sociodemográfico al anegamiento no presenta una diferencia significativa respecto a la del total de la población. Por otro lado, la hipótesis alternativa $\left(\mathrm{H}_{1}\right)$ asume la situación inversa, señalando que el grupo está sujeto a una exposición claramente distinta (mayor o menor) que la población total.

La interpretación de los resultados generados mediante el cálculo de la $\chi^{2}$ (con el software NCSS) permite comprobar la existencia o no de inequidad en cada una de las variables seleccionadas, rechazando $\mathrm{H}_{0}$ cuando el nivel de probabilidad es igual o menor a los niveles de significancia $\alpha=0,01$ o $\alpha=0,05$.

Con la segunda técnica utilizada, la balanza de la justicia ambiental, fue posible observar en qué grado 
FIGURA 3

ÁREA URBANA POBLADA DE SANTA FE CON LAS ZONAS AFECTADAS POR ANEGAMIENTO EN 2010

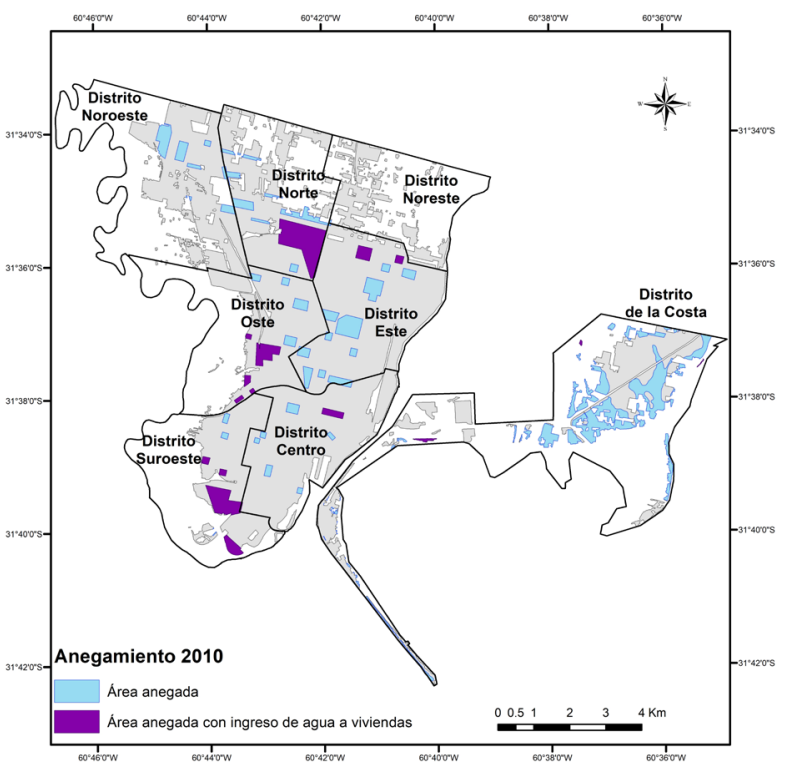

Fuente: elaboración propia en base a datos de MCSF.

(porcentaje) la frecuencia observada para un determinado grupo sociodemográfico, en cada una de las dos categorías (anegada/no anegada), varía respecto de la norma, representada por los porcentajes en el universo de referencia (el conjunto de la ciudad). El procedimiento permite una clara apreciación de la inequidad de cada grupo poblacional al exponer sus resultados en el gráfico de una balanza, clásico e histórico símbolo de la justicia. Su ejecución recurrió al software MS Excel.
FIGURA 4

ÁREA URBANA POBLADA DE SANTA FE CON LAS ZONAS AFECTADAS POR ANEGAMIENTO EN 2015

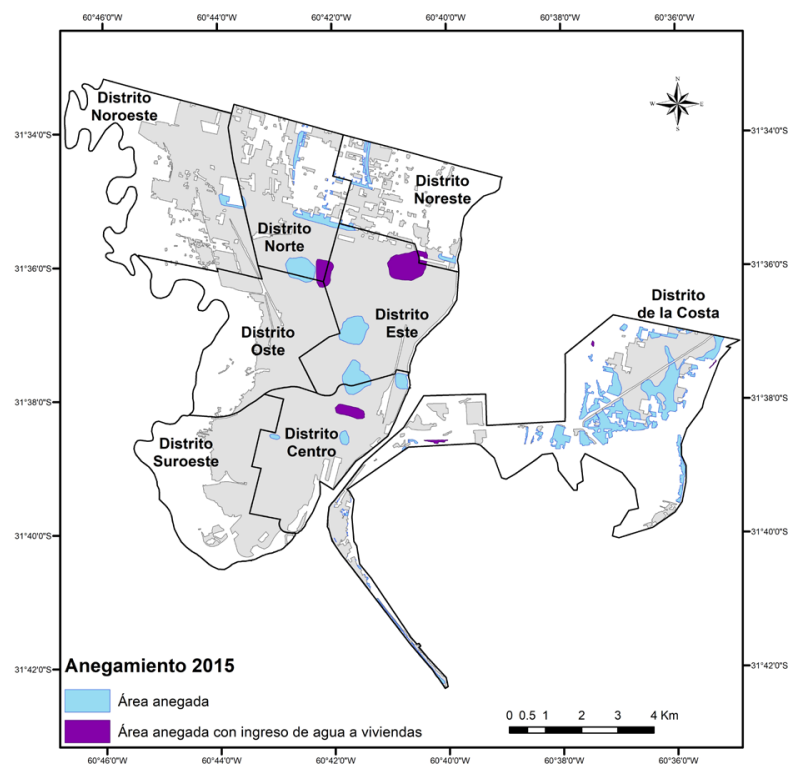

Fuente: elaboración propia en base a datos de MCSF.

En ambas técnicas la metodología de evaluación de la justicia ambiental asume un valor de referencia (criterio base) que varía, de acuerdo al indicador de vulnerabilidad y privación considerado (véase Tabla 1), de modo que una potencial exposición de un grupo determinado al anegamiento que sea proporcionalmente elevada respecto a la del universo de referencia se consideraría como una discriminación y, por ende, una injusticia ambiental.

TABLA 1

VALORES DE REFERENCIA ASUMIDOS PARA LOS PORCENTAJES DE EXPOSICIÓN DE CADA INDICADOR DE VULNERABILIDAD / PRIVACIÓN EN LA EVALUACIÓN DE LA JUSTICIA AMBIENTAL

\begin{tabular}{|c|l|c|c|}
\hline Valor de referencia & \multicolumn{1}{|c|}{ Indicador de vulnerabilidad / grupo de población } & Acrónimo & Dimensión \\
\hline \multirow{2}{*}{ Población total } & Población comprendida entre 0-14 años & PC_0_14 & V \\
\cline { 2 - 4 } & Población comprendida entre 65 y más años & PC_65_MÁS & V \\
\hline $\begin{array}{c}\text { Población de } \\
14 \text { y } 15 \text { años }\end{array}$ & Población comprendida entre 14 y 15 años que trabaja & PC_14_15_T & V-P \\
\hline $\begin{array}{c}\text { Población de } \\
20 \text { a } 24 \text { años }\end{array}$ & $\begin{array}{l}\text { Población comprendida entre } 20 \text { y } 24 \text { años con bajo nivel } \\
\text { educativo }\end{array}$ & PC_20_24_B & V-P \\
\hline \multirow{2}{*}{$\begin{array}{c}\text { Total de viviendas } \\
\text { habitadas }\end{array}$} & Viviendas de calidad constructiva insuficiente & PC_C_I & V-P \\
\cline { 2 - 4 } & Viviendas con conexiones a servicios básicos insuficiente & PC_CNXI & V-P \\
\hline \multirow{2}{*}{\begin{tabular}{c} 
Total de hogares \\
\cline { 2 - 4 }
\end{tabular}} & Hogares con hacinamiento crítico & PC_HOG_HAC & V-P \\
\cline { 2 - 4 } & Hogares con heladera $(*)$ & PC_HOG_HEL & P \\
\cline { 2 - 4 } & Hogares con computadora $(*)$ & PC_HOG_COM & P \\
\hline
\end{tabular}

(*) Por ausencia en el censo de variables alusivas a ese tipo de carencias, se han incluido estos indicadores de dotación (conjunto complementario). Fuente: elaboración propia. 


\section{RESULTADOS}

1. Las magnitudes estimadas de población y superficie afectadas por los anegamientos en la ciudad de Santa Fe en 2010 y 2015

Los anegamientos acaecidos en Santa Fe en el año 2010 fueron visiblemente más extensos y abarcadores que los del año 2015, aunque sus registros poseen cifras similares (Tabla 2); con un porcentaje de superficie anegada en el AUP de $14,58 \%$ y $11,40 \%$ respectivamente. Ello tuvo un correlato en las afecciones sufridas por la población total, las viviendas y los hogares (Tabla 3). Así mismo, esa notable reducción en 2015 se reitera en la afección según los indicadores de privación y vulnerabilidad estudiados (Tabla 4).
2. La desigual discriminación entre los grupos vulnerables y con privaciones por los anegamientos 2010 y 2015

Comenzando con los indicadores de vulnerabilidad y privación que constituyen los grandes grupos de edades, de $\mathbf{0}$ a 14 años (población joven) y de 65 años y más, se puede comprobar que durante el evento de 2010: a) ambos colectivos mostraron grados de exposición notoriamente distintos al de la población total, pues el test de la $\chi^{2}$ arroja una probabilidad cercana a cero; b) tuvieron niveles de afección contrarios, debido a su diferente y opuesto patrón espacial (Tabla 5).

En efecto, tanto los niños como los ancianos presentan en la ciudad de Santa Fe la distribución espacial normalmente observada para estos grupos de edades, los jóvenes con mayor presencia en la periferia y los mayores preferentemente en la zona central.

TABLA 2

DATOS PLUVIOMÉTRICOS Y ALTURA HIDROMÉTRICAS MÁXIMAS OCURRIDAS DURANTE LOS MESES DE LOS ANEGAMIENTOS EN 2010 Y 2015

\begin{tabular}{|c|c|c|c|c|c|c|c|}
\hline Anegamientos & Meses & $\begin{array}{c}\text { Total } \\
\text { mensual de } \\
\text { Iluvia }(\mathrm{mm})\end{array}$ & $\begin{array}{c}\text { Registro } \\
\text { máximo } \\
\text { mensual de } \\
\text { Iluvia (mm) }\end{array}$ & $\begin{array}{l}\text { Media } \\
\text { mensual de } \\
\text { Iluvia }(\mathrm{mm})\end{array}$ & $\begin{array}{c}\text { Total de días } \\
\text { de Iluvia } \\
\text { registrada }\end{array}$ & $\begin{array}{l}\text { Altura } \\
\text { máxima } \\
\text { mensual del } \\
\text { río Paraná } \\
\text { (m) }\end{array}$ & $\begin{array}{l}\text { Altura } \\
\text { máxima } \\
\text { mensual del } \\
\text { río Salado } \\
\text { (m) }\end{array}$ \\
\hline \multirow{2}{*}{2010} & Febrero & 357,30 & 135 & 29,8 & 12 & 6,02 & 4,85 \\
\hline & Marzo & 164,50 & 92,2 & 27,4 & 6 & 5,82 & 4,22 \\
\hline \multirow{2}{*}{2015} & Febrero & 214,25 & 159 & 30,6 & 7 & 4,52 & 5,06 \\
\hline & Marzo & 208,50 & 118,5 & 26,1 & 8 & 4,26 & 6,27 \\
\hline
\end{tabular}

Fuente: Centro de Informaciones Meteorológicas FICH-UNL.

TABLA 3

VALORES DE SUPERFICIE, POBLACIÓN TOTAL, VIVIENDAS Y HOGARES EXPUESTOS A ANEGAMIENTO EN SANTA FE (AUP)

\begin{tabular}{|c|c|c|c|c|c|c|}
\hline Años & $\begin{array}{c}\text { Superficie AUP } \\
\text { (ha) }\end{array}$ & $\begin{array}{c}\text { Superficie } \\
\text { anegada (ha) }\end{array}$ & $\begin{array}{c}\text { \% Superficie } \\
\text { anegada }\end{array}$ & $\begin{array}{c}\text { Población } \\
\text { total en AUP }\end{array}$ & $\begin{array}{c}\text { Población } \\
\text { total } \\
\text { afectada } \\
\text { estimada }\end{array}$ & $\begin{array}{c}\text { Población } \\
\text { total afectada } \\
\text { estimada }\end{array}$ \\
\hline $\mathbf{2 0 1 0}$ & 5792,02 & 844,65 & 14,58 & 391190 & 50605 \\
\hline $\mathbf{2 0 1 5}$ & 5792,02 & 660,48 & 11,40 & 391190 & 34562 & 8,94 \\
\hline
\end{tabular}

\begin{tabular}{|c|c|c|c|c|c|c|}
\hline Años & $\begin{array}{c}\text { Total hogares } \\
\text { en AUP }\end{array}$ & $\begin{array}{c}\text { Total hogares } \\
\text { afectados } \\
\text { estimados }\end{array}$ & $\begin{array}{c}\text { \% Total } \\
\text { hogares } \\
\text { afectados } \\
\text { estimados }\end{array}$ & $\begin{array}{c}\text { Total viviendas } \\
\text { en AUP }\end{array}$ & $\begin{array}{c}\text { Total viviendas } \\
\text { afectadas } \\
\text { estimadas } \\
\text { viviendas } \\
\text { afectadas } \\
\text { estimadas }\end{array}$ \\
\hline $\mathbf{2 0 1 0}$ & 124915 & 15433 & 12,35 & 114897 & 13983 \\
\hline $\mathbf{2 0 1 5}$ & 124915 & 10917 & 8,73 & 114897 & 10038 \\
\hline
\end{tabular}

Fuente: elaboración propia. 
TABLA 4

VALORES Y PORCENTAJES PARA LOS DISTINTOS INDICADORES EN SANTA FE (AUP) AFECTADOS POR LOS ANEGAMIENTOS EN 2010 Y 2015

\begin{tabular}{|c|c|c|c|c|c|c|}
\hline \multirow{2}{*}{ Indicadores } & \multirow{2}{*}{ Total en AUP } & \multirow{2}{*}{$\begin{array}{c}\text { Valor del } \\
\text { indicador en } \\
\text { AUP (\%) }\end{array}$} & \multicolumn{2}{|c|}{ Anegamiento 2010} & \multicolumn{2}{|c|}{ Anegamiento 2015} \\
\hline & & & $\begin{array}{c}\text { Total de } \\
\text { afectados } \\
\text { estimados en } \\
\text { AUP }\end{array}$ & $\begin{array}{c}\% \text { de } \\
\text { afectados } \\
\text { estimados en } \\
\text { AUP }\end{array}$ & $\begin{array}{c}\text { Total de } \\
\text { afectados } \\
\text { estimados en } \\
\text { AUP }\end{array}$ & $\begin{array}{c}\% \text { de } \\
\text { afectados } \\
\text { estimados en } \\
\text { AUP }\end{array}$ \\
\hline P_0_14 & 93461 & 23,89 & 13209 & 14,13 & 8178 & 8,75 \\
\hline P_65_MÁS & 42228 & 10,79 & 4706 & 11,14 & 3692 & 8,74 \\
\hline P_14_15_T & 2384 & 18,12 & 353 & 14,81 & 214 & 8,98 \\
\hline P_20_24_B & 12469 & 35,30 & 1917 & 15,37 & 1134 & 9,09 \\
\hline Viv_C_I & 13834 & 12,04 & 2408 & 17,41 & 1186 & 8,57 \\
\hline Viv_CNXI & 36025 & 31,35 & 6192 & 17,19 & 3729 & 10,35 \\
\hline HOG_HAC & 4094 & 3,28 & 702 & 17,15 & 336 & 8,21 \\
\hline HOG_HEL & 119936 & 96,01 & 14631 & 12,20 & 10525 & 8,78 \\
\hline HOG_COM & 65726 & 52,62 & 7135 & 10,86 & 5651 & 8,60 \\
\hline HOG_NBI & 6910 & 5,53 & 1085 & 15,71 & 570 & 8,25 \\
\hline
\end{tabular}

Fuente: elaboración propia.

La población de 0-14 tiene una mayor densidad en el denominado cordón oeste de la ciudad de Santa Fe, que limita con el cauce principal del Río Salado y su llanura aluvial; así como también en el Distrito Norte y el Distrito de la Costa, lugares en los cuales el anegamiento en 2010 registró su mayor incidencia. Por esta razón, el grupo de población joven resultó marcadamente perjudicado (+1,4 puntos porcentuales) al tener mayor presencia periférica, frente al grupo de población mayor que en este caso, por ubicarse densamente en la zona céntrica de la ciudad, resulta relativamente favorecido (-1,76 puntos porcentuales) (Figura 5 A y B, Tabla 5). En resumen, del análisis se colige una discriminación injusta para los niños y jóvenes.

En el evento ocurrido en el año 2015 no se produjeron anegamientos en dichas áreas denotando el cálculo de la $\chi^{2}$ valores no significativos; por tal motivo la población joven y la población de 65 y más años no fueron excesivamente afectadas y, en consecuencia, los valores revelan una leve subexposición a la amenaza, registrándose $-0,05$ y -0,06 respectivamente (Tabla 6). Esto se grafica claramente en la balanza de justicia ambiental de ambos grupos, de modo tal que las barras quedan muy alineadas horizontalmente, lo cual denota una equi-exposición de estos grupos dentro de la ciudad respecto a la población total (Figura 6 A y B).
Los indicadores correspondientes a las poblaciones de 14 y 15 años que trabaja y de 20 a 24 años con bajo nivel educativo han presentado grados de exposición similares en ambos eventos, resultando en una afección cercana al $15 \%$ para el anegamiento de 2010 y al 9 \% en el anegamiento de 2015 (Tabla 5). El primero de estos dos indicadores denota valores de la $\chi^{2}$ no significativos con una muy leve sobre-exposición en el año 2010, registrando un valor de $+0,41$; mientras que para el evento de 2015 sus valores tampoco reportan diferencias significativas con la ciudad $(+0,28)$, pese a su ubicación espacial mayoritariamente periférica; esto se expresa en sus respectivas balanzas, que poseen una barra en posición casi equilibrada (Figuras 5 C y 6 C).

En cuanto al grupo poblacional representado por personas de 20 a 24 años con bajo nivel de educación, fue significativamente afectado en el evento del año 2010 con un valor de $+2,68$; esto puede deberse a la mayor presencia de este grupo dentro de los barrios marginales, dada su característica sociocultural (Figura 5 D). Así mismo, en el año 2015 también resultó afectado significativamente para la probabilidad $\alpha=0,05$, aunque con solo una leve sobre-exposición al anegamiento $(+0,59)$ (Tabla 6 y Figura 6 D). 
TABLA 5

TEST DE BONDAD DE AJUSTE Y MAGNITUD DE LA AFECCIÓN DIFERENCIAL AL ANEGAMIENTO PARA LOS DISTINTOS INDICADORES EN SANTA FE, 2010

\begin{tabular}{|c|c|c|c|c|c|c|}
\hline Indicadores & $\begin{array}{c}\chi^{2} \text { con 1 } \\
\text { grado de } \\
\text { libertad }\end{array}$ & $\begin{array}{c}\text { Nivel de } \\
\text { probabilidad }\end{array}$ & $\begin{array}{c}\text { Total afectado } \\
\text { (A) }\end{array}$ & $\begin{array}{c}\text { Total de } \\
\text { afectados } \\
\text { esperados } \\
\text { (supuesta } \\
\text { independencia) } \\
\text { (B) }\end{array}$ & $\begin{array}{c}\text { Diferencia: sobre } \\
\text { o subexposición al } \\
\text { anegamiento } \\
\text { (A - B) (*) }\end{array}$ & $\begin{array}{c}\text { Diferencia } \\
\text { (\%): sobre o } \\
\text { subexposición } \\
\text { al anegamiento } \\
\text { (A - B) (*) }\end{array}$ \\
\hline P_0_14 & 118,89 & 0,00 & $13209(14,13 \%)$ & $12090(12,9 \%)$ & 1119 & 1,4 \\
\hline P_65_MÁS & 120,39 & 0,00 & $4706(11,14 \%)$ & $5463(12,9 \%)$ & -757 & $-1,76$ \\
\hline P_14_15_T & 0,398 & 0,528 & $353(14,81 \%)$ & $342(14,4 \%)$ & 11 & 0,41 \\
\hline P_20_24_B & 80,170 & 0,00 & $1918(15,38 \%)$ & $1585(12,7 \%)$ & 333 & 2,68 \\
\hline Viv_C_I & 355,853 & 0,00 & $2409(17,41 \%)$ & $1684(12,2 \%)$ & 725 & 5,21 \\
\hline Viv_CNXI & 848,666 & 0,00 & $6192(17,19 \%)$ & $4384(12,2 \%)$ & 1808 & 4,99 \\
\hline HOG_HAC & 86,824 & 0,00 & $702(17,15 \%)$ & $506(12,4 \%)$ & 196 & 4,75 \\
\hline HOG_HEL & 2,692 & 0,101 & $14631(12,20 \%)$ & $14818(12,4 \%)$ & -187 & $-0,2$ \\
\hline HOG_COM & 136,428 & 0,00 & $7135(10,86 \%)$ & $8120(12,4 \%)$ & -985 & $-1,54$ \\
\hline HOG_NBI & 71,485 & 0,00 & $1085(15,70 \%)$ & $854(12,4 \%)$ & 231 & 3,3 \\
\hline
\end{tabular}

$\left(^{*}\right)$ El signo - significa subexposición. Fuente: elaboración propia.

TABLA 6

TEST DE BONDAD DE AJUSTE Y MAGNITUD DE LA AFECCIÓN DIFERENCIAL AL ANEGAMIENTO PARA LOS DISTINTOS INDICADORES EN SANTA FE, 2015

\begin{tabular}{|c|c|c|c|c|c|c|}
\hline Indicadores & $\begin{array}{c}\chi^{2} \text { con 1 } \\
\text { grado de } \\
\text { libertad }\end{array}$ & $\begin{array}{c}\text { Nivel de } \\
\text { probabilidad }\end{array}$ & $\begin{array}{c}\text { Total afectado } \\
\text { (A) }\end{array}$ & $\begin{array}{c}\text { Total de } \\
\text { afectados } \\
\text { esperados } \\
\text { (supuesta } \\
\text { independencia) } \\
\text { (B) }\end{array}$ & $\begin{array}{c}\text { Diferencia: sobre } \\
\text { o subexposición al } \\
\text { anegamiento } \\
\text { (A - B) (*) }\end{array}$ & $\begin{array}{c}\text { Diferencia } \\
\text { (\%): sobre o } \\
\text { subexposición al } \\
\text { anegamiento } \\
\text { (A - B) (*) }\end{array}$ \\
\hline P_0_14 & 0,858 & 0,354 & $8177(8,75 \%)$ & $8257(8,8 \%)$ & -80 & $-0,05$ \\
\hline P_65_MÁS & 0,444 & 0,505 & $3692(8,74 \%)$ & $3731(8,8 \%)$ & -39 & $-0,06$ \\
\hline P_14_15_T & 0,221 & 0,638 & $214(8,98 \%)$ & $207(8,7 \%)$ & 7 & 0,28 \\
\hline P_20_24_B & 4,897 & 0,027 & $1134(9,09 \%)$ & $1065(8,5 \%)$ & 69 & 0,59 \\
\hline Viv_C_I & 0,463 & 0,496 & $1186(8,57 \%)$ & $1209(8,7 \%)$ & -23 & $-0,13$ \\
\hline Viv_CNXI & 117,791 & 0,00 & $3729(10,35 \%)$ & $3147(8,7 \%)$ & 582 & 1,65 \\
\hline HOG_HAC & 1,455 & 0,228 & $336(8,21 \%)$ & $358(8,7 \%)$ & -22 & $-0,49$ \\
\hline HOG_HEL & 0,195 & 0,659 & $10525(8,78 \%)$ & $10482(8,7 \%)$ & 43 & 0,08 \\
\hline HOG_COM & 1,655 & 0,198 & $5651(8,60 \%)$ & $5744(8,7 \%)$ & -93 & $-0,1$ \\
\hline HOG_NBI & 2,210 & 0,137 & $569(8,23 \%)$ & $604(8,7 \%)$ & -35 & $-0,47$ \\
\hline
\end{tabular}

(*) El signo - significa subexposición. Fuente: elaboración propia. 
FIGURA 5 A, B, CY D

BALANZAS DE LA JUSTICIA AMBIENTAL OBTENIDAS PARA EL ANEGAMIENTO DE 2010

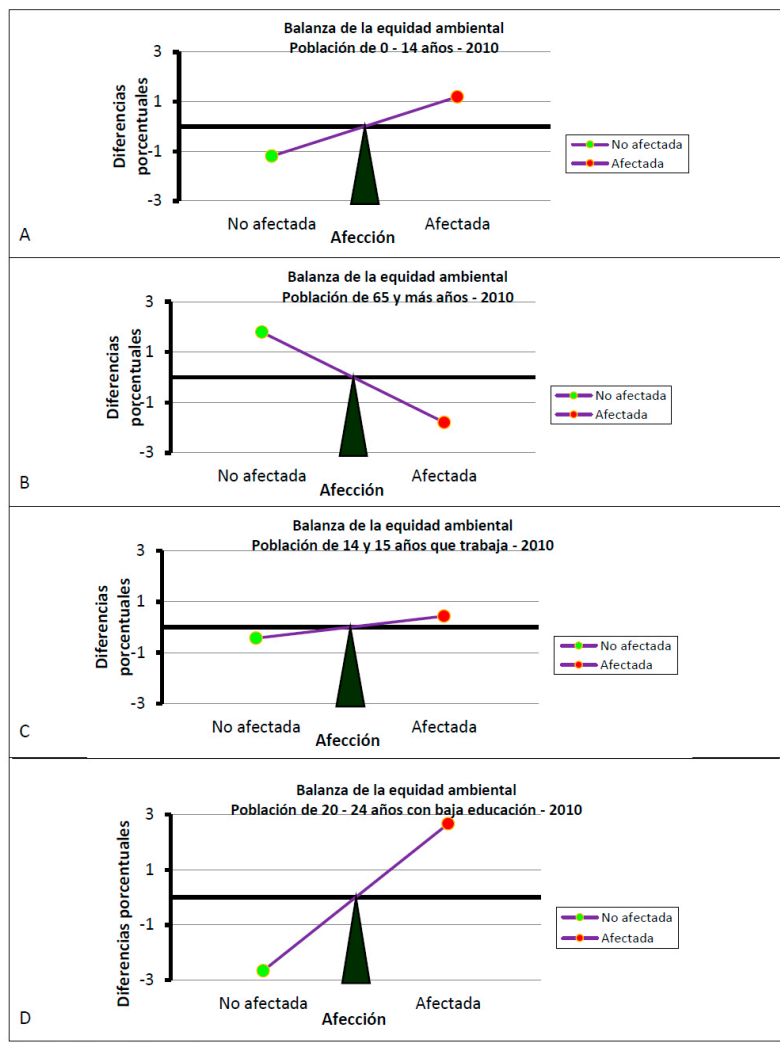

Fuente: elaboración propia.
FIGURA $6 \mathrm{~A}, \mathrm{~B}, \mathrm{C}$ Y D

BALANZAS DE LA JUSTICIA AMBIENTAL OBTENIDAS PARA EL ANEGAMIENTO DE 2015

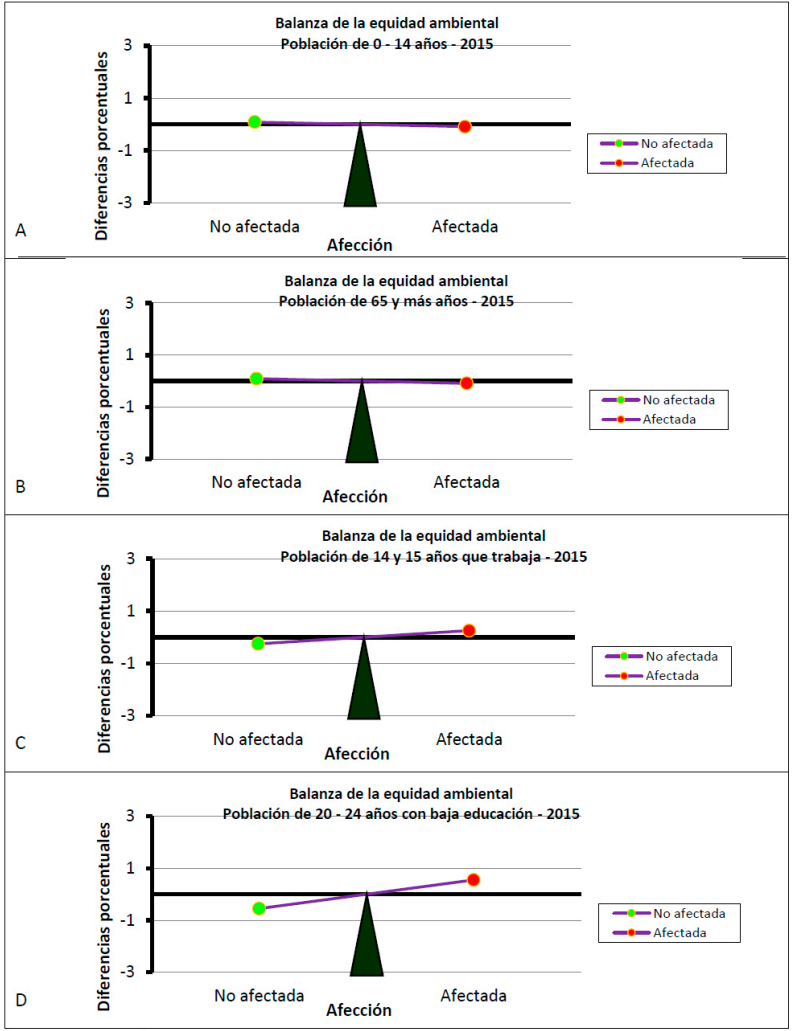

Fuente: elaboración propia.

Esta variación en la afección a las viviendas en ambos períodos de anegamiento tiene su explicación plausible en los cambios estructurales resultantes de la construcción de reservorios y canalizaciones por el gobierno local posteriormente al anegamiento acaecido en el año 2010; ellos aumentaron la capacidad de drenaje específicamente en el cordón oeste de la ciudad, contribuyendo a un mejor y más rápido escurrimiento de las precipitaciones acumuladas y trayendo alivio en el año 2015 a este sector, que fue castigado por las inundaciones provocadas por lluvias intensas en el 2010.

La diferencia de exposición de los hogares con computadora es significativa en 2010 (Tabla 5), demostrando una subexposición respecto al total de la ciudad (-1,54). En 2015 los niveles de afección respecto a los referentes de la ciudad resultan bastante similares, por lo cual el test de la $\chi^{2}$ no resulta significativo en dicho período (Tabla 6 y Figuras 9 A y 10 A). 
FIGURA 7 A Y B

BALANZAS DE LA JUSTICIA AMBIENTAL OBTENIDAS PARA EL ANEGAMIENTO DE 2010

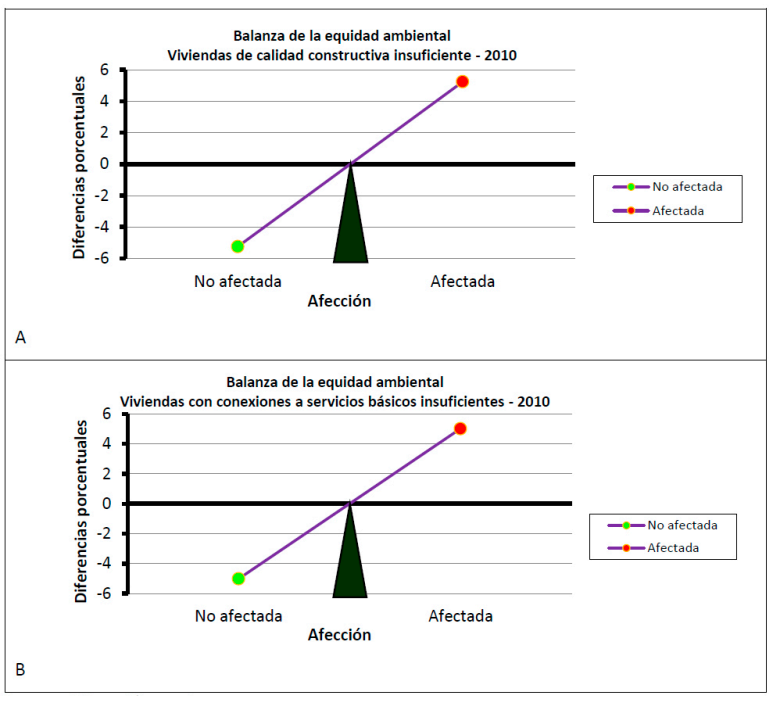

Fuente: elaboración propia.
FIGURA 8 A Y B

BALANZAS DE LA JUSTICIA AMBIENTAL OBTENIDAS PARA EL ANEGAMIENTO DE 2015

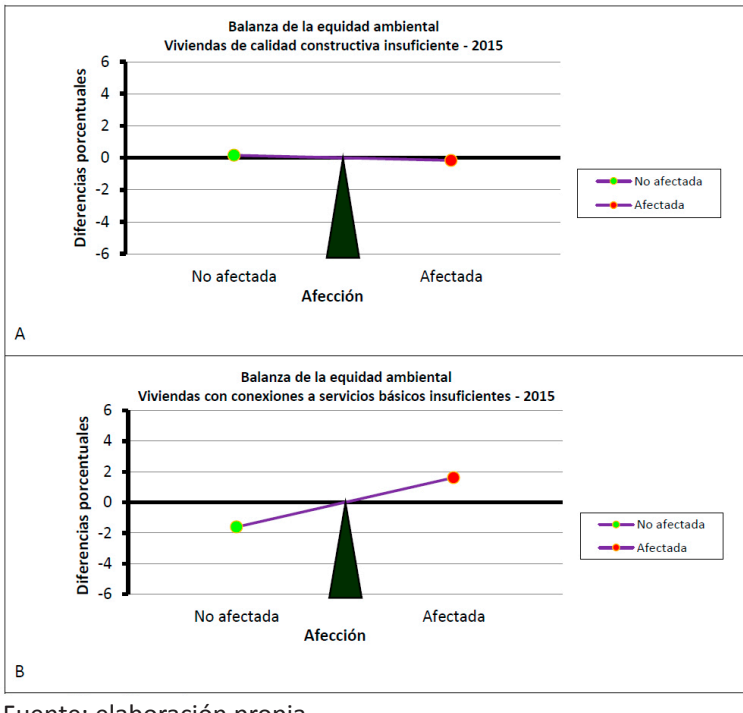

Fuente: elaboración propia.
FIGURA 9 A, B, CY D

BALANZAS DE LA JUSTICIA AMBIENTAL OBTENIDAS PARA EL ANEGAMIENTO DE 2010

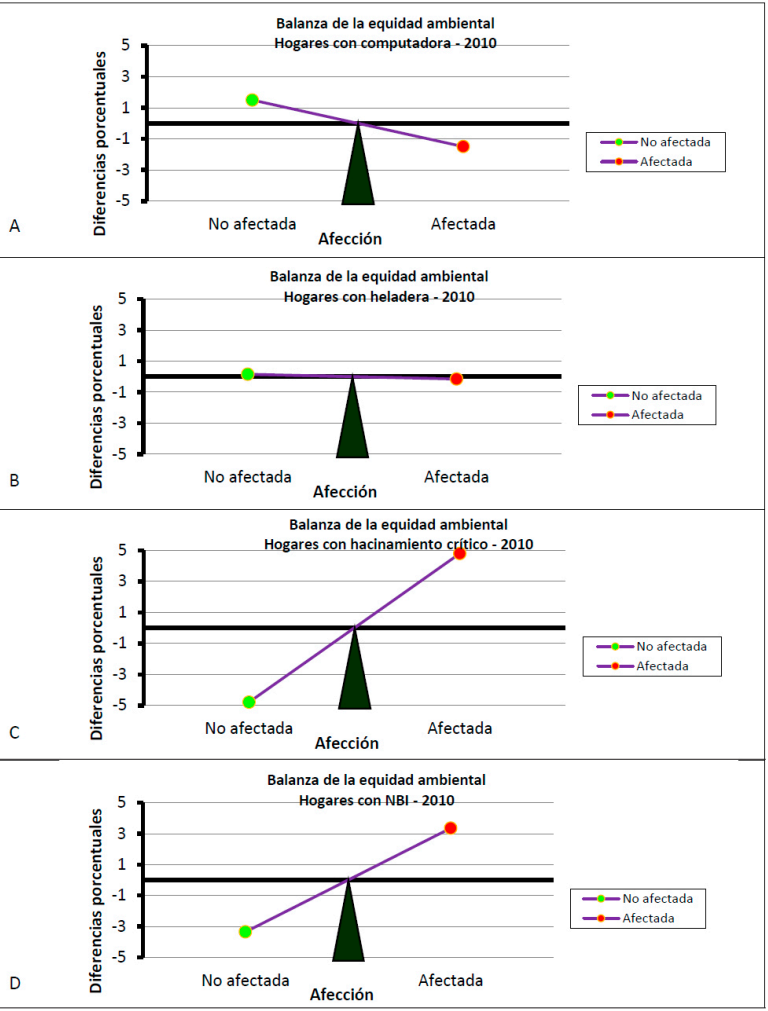

Fuente: elaboración propia.
FIGURA $10 \mathrm{~A}, \mathrm{~B}, \mathrm{C}$ Y D

BALANZAS DE LA JUSTICIA AMBIENTAL OBTENIDAS PARA EL ANEGAMIENTO DE 2015

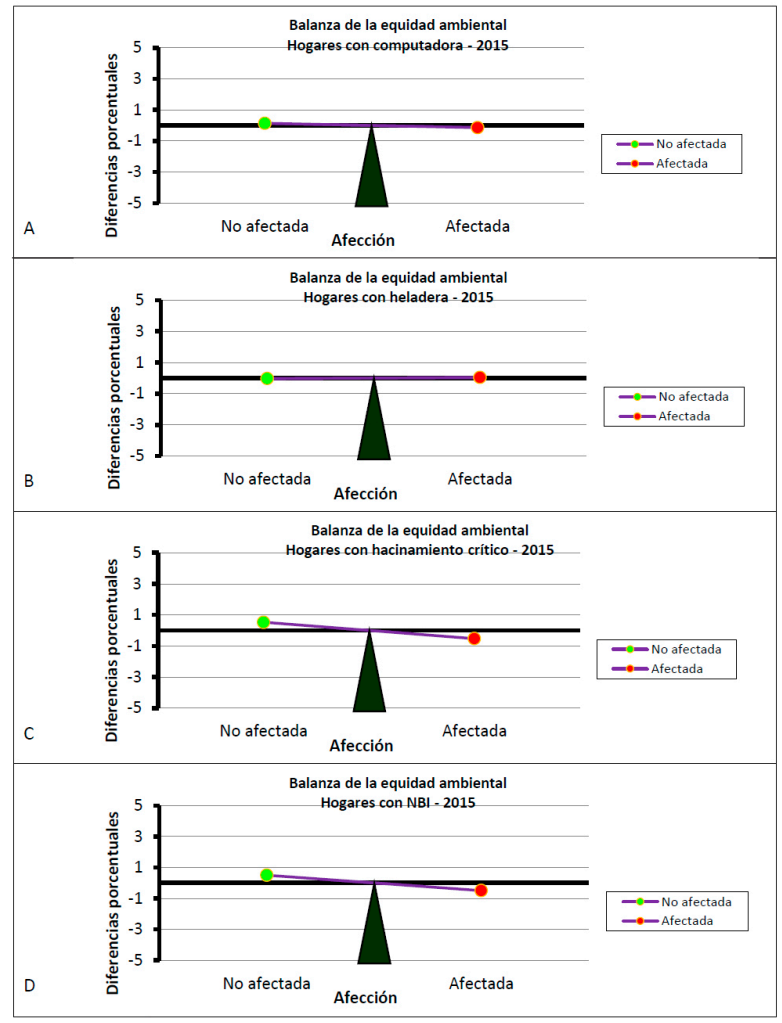

Fuente: elaboración propia. 
Los porcentajes de afección para los hogares con heladera registraron más del $12 \%$ para el año 2010 y casi el $9 \%$ para el año 2015 (Tabla 4). En 2010 este indicador muestra una afección parecida a la del conjunto de la ciudad y el valor de la $\chi^{2}$ tampoco es significativo, ocurriendo una situación similar en el año 2015 (Tablas 5 y 6). De hecho, sus valores reflejan situaciones equilibradas o con un leve grado de sub-exposición a la amenaza ambiental, lo cual muy probablemente se deba a que este electrodoméstico presenta una distribución espacial bastante análoga a la de la población total (Figuras 9 B y 10 B).

El total de hogares con necesidades básicas insatisfechas (NBI) en la ciudad de Santa Fe es de 6910, mayoritariamente ubicados en las zonas periféricas, resultando valores de la $\chi^{2}$ significativos $y$ una marcada sobre-afección $(+3,3)$ para el evento pluvio-hídrico de 2010 (Tabla 5). La situación cambia en el año 2015 (Tabla 6), donde aparece una cierta subexposición $(-0,47)$ para este indicador en la ciudad (Figuras 9 D y 10 D). Esta situación se asemeja mucho a la observada en el grupo de hogares con hacinamiento crítico, fundamentalmente porque ambos grupos exhiben un patrón de distribución intraurbana similar, por lo cual sus valores de exposición a la amenaza ambiental reflejan un paralelismo para ambos períodos (Figuras 9 C y $10 \mathrm{C}$ ).

\section{Análisis de la justicia ambiental para distritos ad- ministrativos por los anegamientos de 2010 y 2015}

La detección de posibles situaciones de injusticias ambientales en cada uno de los ocho distritos administrativos de la ciudad de Santa Fe puede resultar conveniente para una apreciación que facilite la toma de decisiones por esas unidades de gestión.

Para desvelarlas se adaptó la técnica de la balanza de la justicia ambiental y el cálculo de la $\chi^{2}$ como prueba multinomial, utilizando el porcentaje de población total afectada en cada distrito, y teniendo como valor de referencia el porcentaje de la población total afectada para el conjunto de la ciudad.

Planteado como una línea de posible desarrollo ulterior y como ilustración metodológica, el análisis ahora solo se ha aplicado al total de la población y no a subgrupos de ella, como se ha hecho en el apartado previo. Tal extensión sería obviamente factible y estaría justificada, en su caso, por un interés especial en alguno de tales subgrupos.
Los valores de la $\chi^{2}$ obtenidos para el evento pluvio-hídrico de marzo de 2010 son mayormente significativos en todos los distritos, resultando el total de población afectada en la ciudad de 50605 personas (Tabla 3); de ellas el $21 \%$ pertenecía al distrito Norte, casi el $16 \%$ al distrito Suroeste y más del 23 $\%$ al distrito de la Costa (Tabla 7, Figura 11). Esos distritos se ubican en áreas marginales, cercanas al cauce principal de ambos ríos, el Salado al oeste y el Paraná en el este, y por lo tanto son zonas con cotas de niveles bajos. Por ese motivo las cifras de población sobre expuesta a la amenaza ambiental para estos tres distritos fueron elevadas, en especial en el distrito Norte con $8,23 \%$ y el de la Costa, cuya situación fue crítica alcanzando un 10,25\%.

Los cinco distritos restantes de la ciudad de Santa Fe poseen niveles topográficos más elevados y por ello todos los registros de población total afectada son inferiores, lo que genera niveles de subexposición respecto del conjunto urbano, particularmente en el distrito Noreste con -12,59 puntos porcentuales respecto a la ciudad.

En el evento pluvio-hídrico del año 2015 se estimó en 34562 las personas afectadas en la ciudad, siendo el cálculo de la $\chi^{2}$ significativo para todos los distritos. De hecho, el distrito de la Costa no sólo continúa registrando una situación crítica en dicho período, sino que además empeora (en términos relativos) respecto del año 2010, contabilizando un nivel de sobreexposición de 14,35 puntos porcentuales (Tabla 8, Figura 12). En el distrito Norte si bien el panorama aún es comprometido, se da una leve mejoría al llegar a 6,67\%. En tanto el distrito Noreste, que presentaba un escaso grado de afección en el 2010, en 2015 su situación se ve agravada al registrar 2,2 puntos de sobreexposición para su población total. Una situación similar se manifiesta en el distrito Este, que pasa a tener un mayor grado de afección en este segundo período.

En los distritos restantes no se observan mayores inconvenientes en el año 2015, siendo notable la mejoría en los distritos Oeste y Suroeste, los cuales fueron beneficiados con obras de canalización de precipitaciones, realizadas con posterioridad al evento pluviohídrico de 2010, como se mencionó antes. Asimismo, el distrito Noroeste ha reducido considerablemente su nivel de sub-exposición respecto del evento de anegamiento acaecido en 2010. 
TABLA 7

TEST DE BONDAD DE AJUSTE Y MAGNITUD DE LA AFECCIÓN DIFERENCIAL AL ANEGAMIENTO PARA LA POBLACIÓN TOTAL POR DISTRITOS ADMINISTRATIVOS EN SANTA FE, 2010

\begin{tabular}{|c|c|c|c|c|c|c|}
\hline Distritos & $\begin{array}{c}\chi^{2} \text { con 1 grado } \\
\text { de libertad }\end{array}$ & $\begin{array}{c}\text { Nivel de } \\
\text { probabilidad }\end{array}$ & $\begin{array}{c}\text { Población } \\
\text { total afectada } \\
\text { estimada (A) }\end{array}$ & $\begin{array}{c}\text { Población total } \\
\text { esperada (B) }\end{array}$ & $\begin{array}{c}\text { Población } \\
\text { total sobre o } \\
\text { subexpuesta al } \\
\text { anegamiento } \\
\text { (A - B) (*) }\end{array}$ & $\begin{array}{c}\text { \% Población } \\
\text { total sobre o } \\
\text { subexpuesta al } \\
\text { anegamiento } \\
\text { (A - B) (*) }\end{array}$ \\
\hline 1. Noroeste & 692,5522 & 0,00 & $5087(9,18 \%)$ & $7166(12,9 \%)$ & -2079 & $-3,72$ \\
\hline 2.Norte & 3246,3625 & 0,00 & $11507(21,13 \%)$ & $7045(12,9 \%)$ & 4462 & 8,23 \\
\hline 3.Noreste & 1733,0000 & 0,00 & $38(0,31 \%)$ & $1584(12,9 \%)$ & -1546 & $-12,59$ \\
\hline 4.Oeste & 223,2720 & 0,00 & $4843(10,59 \%)$ & $5915(12,9 \%)$ & -1072 & $-2,31$ \\
\hline 5.Este & 12,5035 & 0,00 & $11150(13,35 \%)$ & $10807(12,9 \%)$ & 343 & 0,45 \\
\hline 6.Suroeste & 398,9668 & 0,00 & $7823(15,96 \%)$ & $6339(12,9 \%)$ & 1484 & 3,06 \\
\hline 7.Centro & 3605,5919 & 0,00 & $3173(4,96 \%)$ & $8267(12,9 \%)$ & -5094 & $-7,94$ \\
\hline 8.Costa & 2494,8430 & 0,00 & $6232(23,15 \%)$ & $3482(12,9 \%)$ & 2750 & 10,25 \\
\hline
\end{tabular}

$(*)$ El signo - significa subexposición respecto al conjunto de la ciudad. Fuente: elaboración propia.

TABLA 8

TEST DE BONDAD DE AJUSTE Y MAGNITUD DE LA AFECCIÓN DIFERENCIAL AL ANEGAMIENTO PARA LA POBLACIÓN TOTAL POR DISTRITOS ADMINISTRATIVOS EN SANTA FE, 2015

\begin{tabular}{|c|c|c|c|c|c|c|}
\hline Distritos & $\begin{array}{c}\chi^{2} \text { con 1 grado } \\
\text { de libertad }\end{array}$ & $\begin{array}{c}\text { Nivel de } \\
\text { probabilidad }\end{array}$ & $\begin{array}{c}\text { Población } \\
\text { total afectada } \\
\text { estimada (A) }\end{array}$ & $\begin{array}{c}\text { Población } \\
\text { total } \\
\text { esperada } \\
\text { (B) }\end{array}$ & $\begin{array}{c}\text { Población } \\
\text { total sobre o } \\
\text { subexpuesta al } \\
\text { anegamiento } \\
\text { (A - B) (*) }\end{array}$ & $\begin{array}{c}\text { \% Población } \\
\text { total sobre o } \\
\text { subexpuesta al } \\
\text { anegamiento } \\
\text { (A - B) (*) }\end{array}$ \\
\hline 1. Noroeste & 3344,6360 & 0,00 & $1031(1,86 \%)$ & $4894(8,8 \%)$ & -3863 & $-6,94$ \\
\hline 2.Norte & 3061,6047 & 0,00 & $8476(15,56 \%)$ & $4811(8,8 \%)$ & 3665 & 6,76 \\
\hline 3.Noreste & 71,3325 & 0,00 & $1347(11,00 \%)$ & $1082(8,8 \%)$ & 265 & 2,2 \\
\hline 4.Oeste & 2996,3582 & 0,00 & $718(1,57 \%)$ & $4040(8,8 \%)$ & -3322 & $-7,23$ \\
\hline 5.Este & 4091,6273 & 0,00 & $12628(15,12 \%)$ & $7381(8,8 \%)$ & 5247 & 6,32 \\
\hline 6.Suroeste & 4749,0429 & 0,00 & $0(0,00 \%)$ & $4329(8,8 \%)$ & -4329 & $-8,8$ \\
\hline 7.Centro & 446,7226 & 0,00 & $4130(6,46 \%)$ & $5646(8,8 \%)$ & -1516 & $-2,34$ \\
\hline 8.Costa & 6851,1268 & 0,00 & $6232(23,15 \%)$ & $2378(8,8 \%)$ & 3854 & 14,35 \\
\hline
\end{tabular}

$\left({ }^{*}\right)$ El signo - significa subexposición respecto al conjunto de la ciudad. Fuente: elaboración propia.

\section{BALANCE Y CONCLUSIONES}

La distribución intraurbana de la población en la ciudad de Santa Fe posee un patrón espacial descripto ya por distintos autores (e.g. Cardoso, 2017; Bosisio y Moreno Jiménez, 2020) y asimilable al de otros ámbitos urbanos (Adger, 2006, p. 276). Según él, las condiciones socio habitacionales más favorables y la población más envejecida se registran en la zona central, en contraposición a la periferia (zonas oeste, norte y este costero), en la cual se ubican los radios censales con mayores carencias dotacionales y residentes en condiciones sociales más vulnerables, desfavorecidas y jóvenes.
El análisis aquí realizado sobre la desigual exposición al anegamiento en el año 2010, para los indicadores considerados, evidenció un patrón espacial característico: de manera clara y consistente los distritos administrativos con cargas ambientales más elevadas se ubicaban preferentemente en el "cordón límite oeste" (que se extiende entre los distritos Suroeste, Oeste y Noroeste), el distrito de la Costa, el distrito Este y el sector sur del distrito Norte. Consecuentemente los ámbitos que sufrieron mayor inequidad (por excesiva afección) en ambos períodos de anegamientos fueron el distrito de la Costa y el distrito Norte, destacándose también con el mismo padecimiento en el año 2010 el distrito Suroeste, y en el año 2015 los distritos Este y Noreste. 
FIGURA 11

MAPA CON LAS BALANZAS DE EQUIDAD AMBIENTAL PARA LA POBLACIÓN TOTAL AFECTADA ESTIMADA EN CADA DISTRITO DURANTE LOS ANEGAMIENTOS DE 2010 EN SANTA FE

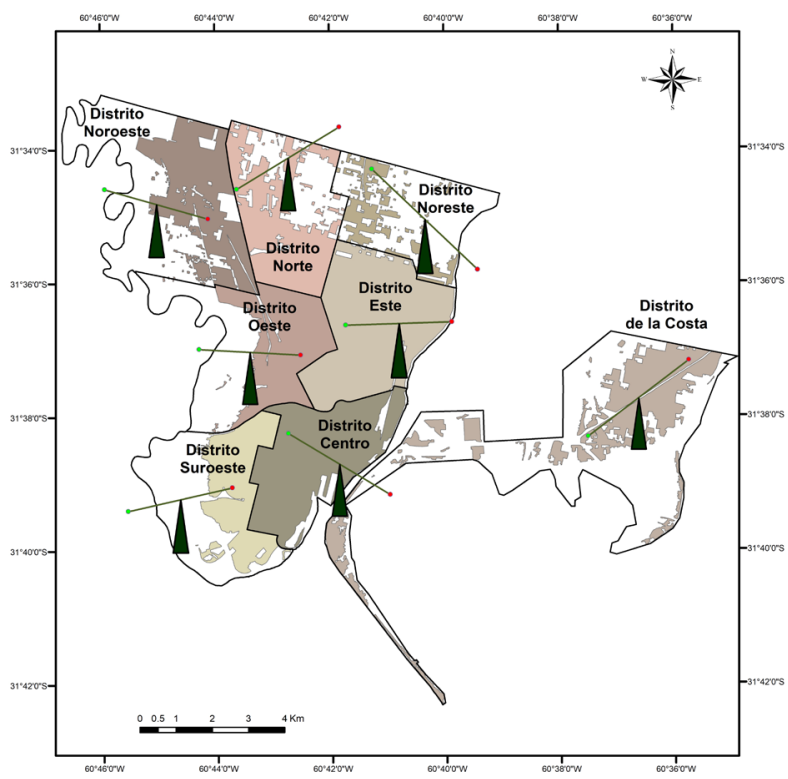

Fuente: elaboración propia.
FIGURA 12

MAPA CON LAS BALANZAS DE EQUIDAD AMBIENTAL PARA LA POBLACIÓN TOTAL AFECTADA ESTIMADA EN CADA DISTRITO DURANTE LOS ANEGAMIENTOS DE 2015 EN SANTA FE

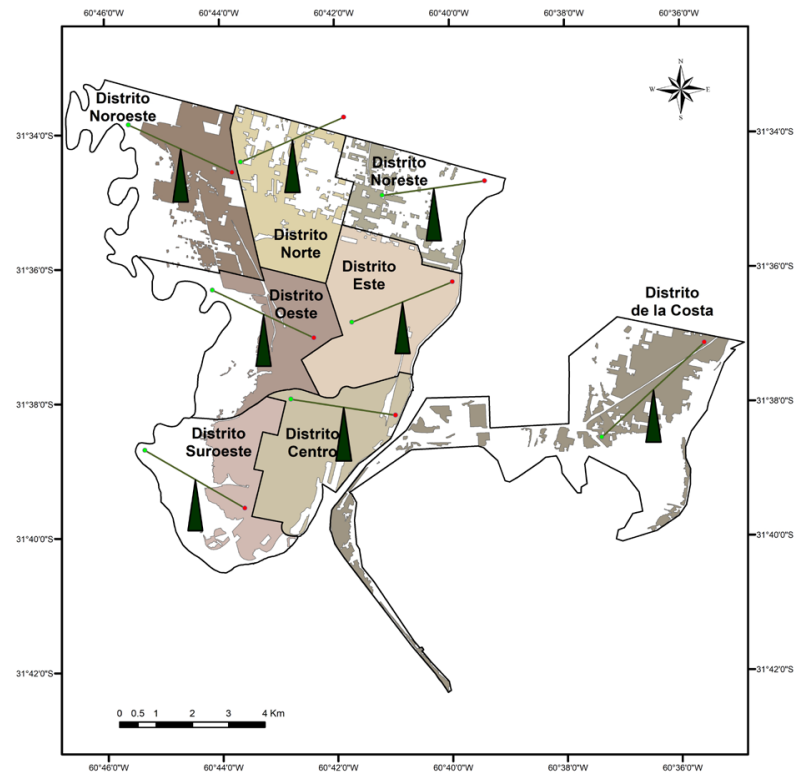

Fuente: elaboración propia.
Los indicadores sociodemográficos que, para el año 2010, revelaron mayores inequidades ambientales fueron seis: la población joven, la población de 20 a 24 años con bajo nivel educativo, las viviendas de calidad constructiva insuficiente, las viviendas con conexiones a servicios básicos insuficiente, los hogares con NBI y los hogares con hacinamiento crítico. Ello pone de manifiesto la seriedad del problema en dicho evento, pues sobre-penalizó a una importante serie de grupos de población desfavorecida. Por el contrario, para el anegamiento ocurrido en el año 2015 solamente dos de los diez indicadores desvelaron desigualdades injustas: la población de 20 a 24 años con bajo nivel educativo y las viviendas con conexiones a servicios básicos insuficiente. Estos resultados permiten aseverar que en el primer período de anegamiento los sectores donde prevalecen grupos con varios tipos de vulnerabilidad y privaciones fueron mayormente anegados; en contraposición a ello, en el evento del año 2015 la sobre-penalización solo afectó a dos categorías de desfavorecidos, lo que implica una mejoría notable.

Procede añadir que los resultados de la afección provocada por el anegamiento del año 2015 reflejan también un cambio en el patrón espacial, pues los sectores de los distritos que pertenecen al cordón oeste ya no se encuentran afectados, probablemente debido a la incidencia de las obras que aumentaron la capacidad de escurrimiento en dicho sector. Ello ocasionó una mejoría para los grupos más vulnerables y con privaciones. Por otro lado, los radios censales con mayor carga ambiental se mantienen constantes en el distrito de la Costa y en el sector sur del distrito Norte, aumentando su número en el distrito Este. Ello pone de manifiesto una imperiosa necesidad de obras que alivien la situación en dichos espacios de cara a futuros anegamientos que se produzcan por similares condiciones pluvio-hídricas.

Con una perspectiva más amplia y comparativa cabe señalar que el patrón espacial de afección hallado en este estudio guarda una cierta similitud y coincidencia con el patrón de vulnerabilidad social y ambiental delimitado precedentemente por otros autores para la ciudad de Santa Fe (Arrillaga et al., 2009, p. 92-96; Cardoso, 2017, p. 172). Es de destacar, sin embargo, que esas investigaciones se realizaron tomando la totalidad de las hectáreas correspondientes a los radios censales, la cual incluye no pocas zonas inhabitadas pertenecientes a espacios rurales, 
islas, y a la planicie de inundación de los ríos Salado y Paraná, con la consiguiente inexactitud derivada. En el presente trabajo, el uso de una delimitación más específica, el área urbana poblada, posibilitó no solo identificar y medir las zonas de afección con mayor rigor espacial, sino además obtener una mejor estimación cuantitativa de la población afectable y de las inequidades que conciernen a los grupos vulnerables y carenciados. En consonancia con diversos autores (Walker and Burningham, 2011; Qiang, 2019) este trabajo ha proporcionado evidencias métricas claras y sistemáticas de las injusticias ambientales ocasionadas por inundaciones urbanas.

La metodología adoptada, apoyada en SIG, se ha mostrado eficaz para los fines perseguidos, por lo que puede recomendarse su utilización para investigar el alcance de dichas injusticias en las labores de planificación y gestión de riesgos de inundación. Ello ha sido propugnado y respaldado ya por diversos au-

\section{Biblografía}

Adger, W. N. (2006). Vulnerability. Global environmental change, 16(3), 268-281. https://doi. org/10.1016/j.gloenvcha.2006.02.006

Aragón-Durand, F. (2014). Inundaciones en zonas urbanas de cuencas en América Latina. Lima, Perú, Editorial Soluciones Prácticas. Recuperado de: http:// www.solucionespracticas.org.pe/Inundacionesen-zonas-urbanas-de-cuencas-en-America-Latina

Arrillaga, H., Grand, L. y Busso, G. (2009). Vulnerabilidad, riesgo y desastres. Sus relaciones de causalidad con la exclusión social en el territorio urbano santafesino. En: Herzer, $\mathrm{H}$. y Arrillaga, $\mathrm{H}$. (coords.). La construcción social del riesgo y el desastre en el aglomerado Santa Fe. $1^{\circ}$ edición, (pp. 59-104). Santa Fe, Argentina: Universidad Nacional del Litoral.

Atuesta Montes, B., Mancero, X. y Tromben Rojas, V. (2018). Herramientas para el análisis de las desigualdades y del efecto redistributivo de las políticas públicas. Comisión Económica para América Latina y el Caribe (CEPAL), Naciones Unidas.

Birkmann J. (2006). Measuring vulnerability to natural hazards: Towards disaster resilient societies. Tokyo: United Nations University Press.

Blaikie, P.M., Cannon, T., Davis, I., Wisner, B. and Blaikie, P. (1994). At risk: natural hazards, people's vulnerability, and disaster. New York: Routledge. tores (e.g. Walker, 2012; Martynoga, 2018; Paloniemi et al., 2018), puesto que posibilitan obtener mapas de peligros y siniestros que, superpuestos a los datos demográficos, identifican más nítidamente quiénes, cuánto y dónde se sufren las consecuencias de estos traumáticos eventos.

\section{AGRADECIMIENTOS}

A la Facultad de Ingeniería y Ciencias Hídricas - UNL, por el apoyo recibido para la realización del presente trabajo. A la Municipalidad de la ciudad de Santa Fe, al Instituto Provincial de Estadísticas y Censos de la Provincia de Santa Fe (IPEC-INDEC), y al Instituto Geográfico Nacional, por facilitar generosamente los datos utilizados en los diversos análisis. Al Departamento de Geografía de la Universidad Autónoma de Madrid por su cordial acogida durante la realización de esta investigación. A los dos revisores anónimos cuyos comentarios contribuyeron a enriquecer el presente trabajo.

Bosisio, A. y Moreno Jiménez, A. (2020). Análisis espacial de indicadores de vulnerabilidad y privaciones sociales basado en SIG: el caso de Santa Fe de la Vera Cruz (Argentina). Geografía y Sistemas de Información Geográfica (GEOSIG), 17, (en prensa).

Cardoso, M. (2017). Estudio de la vulnerabilidad socio-ambiental a través de un índice sintético. Caso de distritos bajo riesgo de inundación: Santa Fe, Recreo y Monte Vera, Provincia de Santa Fe, Argentina. Caderno de Geografia, 27(48),156-183. Pontifícia Universidad Católica de Minas Gerais, Belo Horizonte, Brasil. https://doi.org/10.5752/ p.2318-2962.2017v27n48p156

Chakraborty, J. (2017). Focus on environmental justice: new directions in international research, Environmental Research Letters, 12(3), 030201. https://doi.org/10.1088/1748-9326/aa63ff

Cutter, S.L., Boruff, B.J. and Shirley, W.L. (2003). Social vulnerability to environmental hazards. Social Science Quarterly, 84(2), 242-261.

Cutter, S.L., Mitchell, J.T. and Scott, M.S. (2000). Revealing the vulnerability of people and places: a case study of Georgetown County, South Carolina. Annals of American Geographers, 90(4), 713-737.

Egea Jiménez, C., Sánchez González, D. y Soledad Suescún, J. (2012). Vulnerabilidad social: posicionamiento y ángulos desde geografías diferen- 
tes. Granada (España): Editorial Universidad de Granada.

Hsu, W.K., Huang, P.C., Chang, C.C., Chen, C.W., Hung, D.M. and Chiang, W.L. (2011). An integrated flood risk assessment model for property insurance industry in Taiwan. Environmental Hazards, 58(3), 1295-1309.

Instituto Nacional de Estadísticas y Censos (INDEC). (2013). Censo Nacional de Población, Hogares y Viviendas 2010: Censo del Bicentenario. Base de datos REDATAM: Definiciones de la base de datos. Serie Base de datos censo 2010. Buenos Aires, Argentina.

Maguire, K. and Sheriff, G. (2011). Comparing distributions of environmental outcomes for regulatory environmental justice analysis. International Journal of Environmental Research and Public Health, 8 (5), 1707-1726.

Martynoga, C. (2018). The Environmental Justice Implications of the Planning Policy and Practice of Flood Risk Management in New Zealand. Thesis Doctoral. Faculty of Arts \& Social Sciences, The University of Waikato Hamilton, New Zealand.

Mendenhall, W., Beaver, R. y Beaver, B. (2010). Introducción a la probabilidad y estadística. México, 13a ed.: Cengage Learning Editores.

Moreno Jiménez, A. (2010). Justicia ambiental. Del concepto a la aplicación en análisis de políticas y planificación territoriales. Scripta Nova, XIV (316). Recuperado de http://www.ub.es/geocrit/sn/sn316.html

Moreno Jiménez, A. (2013). Población y polución atmosférica intraurbana por dióxido de nitrógeno en Madrid: análisis desde la justicia ambiental basado en sistemas de información geográfica. Cuadernos Geográficos de la Universidad de Granada, 52 (1), 84-107. Recuperado de http://revistaseug.ugr.es/ index.php/cuadgeo/article/download/927/1078

Moreno Jiménez, A., Buzai, G. y Fuenzalida, M. (Coords.) (2017). Sistemas de Información Geográfica: aplicaciones en diagnósticos territoriales y decisiones geoambientales. Madrid: Ra-Ma, 2a ed.

Moreno Jiménez, A., Cañada-Torrecilla, R., Vidal-Domínguez, M.J., Palacios-García, A. and MartínezSuárez, P. (2016). Assessing environmental justice through potential exposure to air pollution: A socio-spatial analysis in Madrid and Barce-
Iona, Spain. Geoforum, 69, 117-131. https://doi. org/10.1016/j.geoforum.2015.12.008

Moreno Jiménez, A., Palacios García, A. J. y Mellado San Gabino, A. (2014a). Evaluación de la justicia ambiental por distritos urbanos como base para un nuevo desarrollo. El caso de Barcelona. En: Serrano Rodríguez, A. (ed.): Patrimonio y planificación territorial como instrumentos para otro desarrollo. Relatoría, ponencias y comunicaciones. VII Congreso Internacional de Ordenación del Territorio. Madrid: FUNDICOT, 1035-1046.

Moreno Jiménez, A., Vidal Domínguez, M. J. y Cañada Torrecilla, R. (2014b). Evaluación de la justicia ambiental por distritos urbanos como base para las políticas y la intervención públicas. Actas del XVI Congreso Nacional de Tecnologías de la Información Geográfica, Universidad de Alicante y Grupo de Tecnologías de la Información Geográfica (AGE), Alicante, pp. 144-152.

Palacios García A., Mateos Rodríguez P., Hidalgo Giralt, C. (2018). A theoretical and methodological essay on the concept of vulnerability, Contexto, XIII(17), 109-122.

Paloniemi, R., Niemelä, J., Soininen, N., Laatikainen, T., Vierikko, K., Rekola, A., Viinikka, A., Yli-Pelkonen, V., Assmuth, T., Kopperoinen, L., Peltonen, L., Kuokkanen, T. and Kyttä, M. (2018). Environmental justice for the governance of aquatic environments. Local Environment, 23(3), 366-377. https://doi.org /10.1080/13549839.2017.1422118

Pandey, A.C., Singh, S.K. and Nathawat, M.S. (2010). Waterlogging and flood hazards vulnerability and risk assessment in Indo Gangetic plain. Natural Hazards, 55(2), 273-289. https://doi. org/10.1007/s11069-010-9525-6

Paoli, C., Dondeynaz, C. y Carmona-Moreno, C. (2015). Gestión integrada de crecidas: guía y caso de estudio. European Commission, Joint Research Centre. Luxembourg: Publications Office of the European Union. https://doi.org/10.2788/997460

Qiang, Y. (2019). Disparities of population exposed to flood hazards in the United States. Journal of Environmental Management 232, 295-304. https:// doi.org/10.1016/j.jenvman.2018.11.039

Rufat, S., Tate, E., Burton, C. and Maroof, A. (2015). Social vulnerability to floods: Review of case studies and implications for measurement. International Journal of Disaster Risk Reduction, 14, 470-486. https://doi.org/10.1016/j.ijdrr.2015.09.013 
Sánchez González, D. y Chávez Alvarado, R. (2016). Personas mayores con discapacidad afectadas por inundaciones en la ciudad de Monterrey, México. Análisis de su entorno físico-social, Cuadernos Geográficos, 55(2), 85-106. Recuperado de http:// www.redalyc.org/articulo.oa?id=17149048004

Sarma, A., Singh, V., Kartha, S. and Bhattacharjya, R. (Eds.). (2016). Urban hydrology, watershed management and socio-economic aspects. Switzerland: Springer https://doi.org/10.1007/978-3319-40195-9

Schneiderbauer S., Calliari E., Eidsvig U., Hagenlocher M. (2017). The most recent view of vulnerability, En: Poljanšek K., Marin Ferrer M., De Groeve T., Clark I., Eds., Science for disaster risk management 2017. Knowing better and losing less. EUR 28034. Luxembourg: Publications Office of the European Union, 68-82.

Siegel, S. (1976). Estadística no paramétrica aplicada a las ciencias de la conducta. México: Editorial Trillas.

Sultana, F. (2010). Living in hazardous waterscapes: Gendered vulnerabilities and experiences of floods and disasters. Environmental Hazards, 9, 43-53. https://doi.org/10.3763/ehaz.2010.SI02

Swim, J. and Bloodhart, B. (2018). The intergroup foundations of climate change justice. Group Pro- cesses \& Intergroup Relations, 21(3), 472-496. https://doi.org/10.1177/1368430217745366

Török, I. (2018). Qualitative Assessment of Social Vulnerability to Flood Hazards in Romania. Sustainability, 10, 3780. https://doi.org/10.3390/ su10103780

Towers, G. (2000). Applying the political geography of scale: the grassroots strategies and environmental justice. The Professional Geographer, 52 (1), 23-36. https://doi.org/10.1111/0033-0124.00202

Townsend, P. (1987). Deprivation, Journal of Social Policy, 16(2), 125-146. https://doi.org/10.1017/ S0047279400020341

Tucci, C. (2007). Gestión de inundaciones urbanas. IPH/UFRGS - ABRH - OMM. Porto Alegre, Brasil: Instituto Superior de Recursos Hídricos, y Argentina: Universidad Nacional de Córdoba, Recuperado de: http://www.apfm.info/pdf/Urban_Flood_ Management_Es_low.pdf

Walker, G. (2012). Environmental justice: concepts, evidence and politics. London, Routledge.

Walker, G. and Burningham, K. (2011). Flood risk, vulnerability and environmental justice: evidence and evaluation of inequality in a UK context. Critical Social Policy, 31(2), 216-240. https://doi. org/10.1177/0261018310396149 